\title{
Comparison of Quantitative Parameters in Diabetics Without Clinical Signs of Diabetic Retinopathy and Healthy Individuals Using Swept-source Optical Coherence Tomography Angiography
}

\section{Sophie Beka}

Kepler University Hospital

Dominika Podkowinski

Kepler University Hospital

Jascha Wendelstein

Kepler University Hospital

Anna-Sophie Mursch-Edlmayr

Kepler University Hospital

Siegfried Mariacher

Kepler University Hospital

Matthias Bolz ( $\square$ matthias.bolz@kepleruniklinikum.at )

Kepler University Hospital

\section{Research Article}

Keywords: Angiography, Swept-source, diabetic retinopathy, Optical Coherence Tomography

Posted Date: January 6th, 2021

DOl: https://doi.org/10.21203/rs.3.rs-133250/v1

License: (c) (i) This work is licensed under a Creative Commons Attribution 4.0 International License.

Read Full License 
Comparison of quantitative parameters in Diabetics without clinical signs of diabetic retinopathy and healthy individuals using Swept-source Optical Coherence Tomography Angiography

Sophie Beka MD ${ }^{1}$, Dominika Podkowinski MD ${ }^{1}$, Jascha Wendelstein MD ${ }^{1}$, AnnaSophie Mursch-Edlmayr MD ${ }^{1}$, Siegfried Mariacher MD ${ }^{1}$, Matthias Bolz MD ${ }^{1,2}$

Corresponding author: Univ.-Prof. Dr. Matthias Bolz, Department of Ophthalmology and Optometry, Kepler University Hospital, Krankenhausstrasse 9, 4020 Linz, Austria.

Email: Matthias.bolz@kepleruniklinikum.at

Phone: +43 (0)5 7 680830-1020

Fax: $+43(0) 5768083-1822$

${ }^{1}$ Johannes Kepler University Linz, Kepler University Hospital GmbH, Department for Ophthalmology and Optometry, Linz 4020, Austria

2 Johannes Kepler University, Medical Faculty, Linz, Austria 


\section{Abstract}

Automated image analysis is the future for retinal imaging in order to early diagnose retinal diseases like diabetic retinopathy. Swept source coherence tomography angiography offers new insights in retinal vascularization. Data sets for each stage of diabetic retinopathy are crucial to compare findings to the healthy population and to develop further algorithms. We recruited 39 eyes of Type-2 diabetics without diabetic retinopathy comparing them to 43 eyes of age and gender matched individuals using swept source coherence tomography angiography. Vessel and perfusion density in the superficial and deep retinal plexus, as well foveal-avascular zone expansion, using Macular Density V 0.7 algorithm were evaluated. We found no significant change in vessel and perfusion density in both plexus, as well no difference in the foveal-avascular zone between healthy and diabetic eyes without clinical diabetic retinopathy.

Current parameters of vascular analysis used in swept- source optical coherence tomography angiography are not valid to detect possible early, subclinical changes in patients with diabetes. However, the presented data set is of high value for future studies, as is represents a complete and clean data set. As automated image analysis is the future in diabetic retinopathy the presented data will be used in future studies. 


\section{Introduction}

Diabetes mellitus (DM) is a widespread disease affecting 366 million people worldwide ${ }^{1}$. Diabetic retinopathy (DR), a complication of DM, is the leading cause of vision loss amongst working age adults in developed countries ${ }^{2}$.

Optical coherence tomography angiography (OCTA) is a novel imaging technique using decorrelation between resampled images to detect blood-flow and thus, highlight 2- and 3-dimensional reconstructions of the retinal vasculature ${ }^{3}$.

Ultra-high speed swept-source optical coherence tomography (SS-OCT) with its higher wavelength and thus its deeper penetration into tissue coupled with the new modality of OCTA, identifies superficial and deep vascular retinal plexus noninvasively ${ }^{4,5}$.

Automated image analysis is the future of retinal imaging. There are multiple studies using OCT or fundus images and creating algorithms based on classic machine learning or deep neural networks ${ }^{6} 7$. Especially in DR there are multiple algorithms available to detect DR changes ${ }^{8}$.

The same image analysis methods can be used for the analysis of OCTA data. As this technique is rather new, there is less data published compared to conventional OCT. However, it was demonstrated that OCTA can be used for DR differentiation ${ }^{9}$ 10. In the future screening methods using artificial intelligence will be of high importance ${ }^{11}$. In order to develop robust algorithms clean data sets are crucial.

Especially in the early stage of DM it is of great interest to investigate retinal microvasculature on its presence of early subclinical changes using automated algorithms. Therefor clean data sets of retinal microvasculature in diabetic patients without clinical signs of DR are needed for different SS-OCTA devices as results 
regarding quantitative changes in retinal microvasculature are almost impossible to compare between different SS-OCAT devices ${ }^{12}$.

The aim of this study was to examine quantitative parameters of retinal microvasculature in diabetic patients without clinical signs of DR using SS-OCTA and comparing them to healthy volunteers. We present a complete data set of diabetic patients without DR and age-matched healthy controls. 


\section{Results}

Thirty-nine eyes of 26 patients (48.1\%) with Type-2 diabetes and 43 eyes of 28

(51.9 \%) healthy individuals were included. All participants were Caucasian.

Table 1 shows demographic and clinical data as well as medication of diabetic patients. Table 2 shows demographic and clinical data of healthy participants.

\begin{tabular}{|l|l|}
\hline Demographic Values & Diabetics (n=26) \\
\hline Women, No. (\%) & $13(50)$ \\
\hline Age, median [IQR], range, years & $69(41-91)$ \\
\hline Clinical Values & \\
\hline Diabetes duration, mean [SD], years & $5.7( \pm 3.4)$ \\
\hline Hba1c, mean [SD], \% & $7.8( \pm 1.2)$ \\
\hline Arterial hypertension, No. (\%) & $19(73.1)$ \\
\hline Medication & \\
\hline One oral antidiabetic, No. (\%) & 14 \\
\hline Two oral antidiabetic, No. (\%) & 9 \\
\hline Three oral antidiabetic, No. (\%) & 1 \\
\hline Short term insulin, No. (\%) & 2 \\
\hline No medication, No. (\%) & 1 \\
\hline Metformin 1000mg, No. (\%) & 18 \\
\hline Metformin 850mg, No. (\%) & 3 \\
\hline Gliclazid 30 mg, No. (\%) & 3 \\
\hline Empaglifozin 10 mg, No. (\%) & 2 \\
\hline Linagliptin, No. (\%) & 1 \\
\hline Vildagliptin, No. (\%) & 5 \\
\hline Sitagliptin, No. (\%) & 1 \\
\hline
\end{tabular}

Table 1 Demographic and clinics values and medication of diabtic patients.

* No. (number), IQR (Interquartile range), CI (Confidence interfvall), SD (standard deviation)

\begin{tabular}{|l|l|}
\hline Demographic Values & $\begin{array}{l}\text { Healthy } \\
\text { participants } \\
\text { (n=28) }\end{array}$ \\
\hline Women, No. (\%) & $14(50)$ \\
\hline Age, median [IQR], range, years & $71(45-85)$ \\
\hline Clinical Values & \\
\hline Arterial hypertension, No. (\%) & $11(39.3)$ \\
\hline
\end{tabular}

Table 2 Demographic and clinical values of the healthy participants.

*No (number), IQR (Interquartile range) 
Regarding age no significant difference could be found between the groups $(p=0.354)$.

FAZ size of both groups was analyzed. Mean (SD) FAZ size in the nondiabetic group was $0.228( \pm 0.137) \mathrm{mm}^{2}$ and in the diabetic group $0.250( \pm 0.122) \mathrm{mm}^{2}(p=0.439$, unpaired t-test).

Examining the SRL of nondiabetic eyes the mean (SD) PD was $0.334( \pm 0.030)$ and mean (SD) VD was $14.838( \pm 1.433)$.

In diabetic eyes the mean (SD) PD was $0.339( \pm 0.033)$ and mean $(S D)$ VD was $14.968( \pm 1.638)$. Regarding a difference in both parameters, neither PD $(p=0.480)$, nor VD $(p=0.701)$ showed a statistically significant difference in the SRL when comparing nondiabetic eyes to eyes of diabetic patients without clinical signs of DR. Within the DRL of healthy eyes the mean (SD) PD was $0.214( \pm 0.050)$ and mean (SD) VD was $10.186( \pm 2.303)$. Among the diabetic eyes mean (SD) PD was 0.228 $( \pm 0.051)$ and mean $(S D)$ VD was $10.734( \pm 2.318)$. Again, neither PD $(p=0.249)$, nor VD $(p=0.291)$ showed a significant difference in the DRL between healthy eyes compared to eyes of diabetic patients without clinical signs of DR. 


\section{Discussion}

In this study we compared quantitative parameters of the retinal microvasculature of patients suffering from diabetes without clinical signs of DR to non-diabetic participants using SS-OCTA.

The rational was, if the use the deeply penetrating SS-OCTA was able to reveal first preclinical changes in diabetic patients without DR before clinically mild NPDRP arise. Our study showed no significant difference in PD and VD, neither in the SRL, nor in the DRL between nondiabetic individuals and diabetic patients without clinical DR.

Automated image analysis is the future of retinal imaging. Clean data sets are crucial for different OCTA devices especially in the early stage of the disease to diagnose first microvascular changes before complications arise.

We present a complete and detailed data set of patients with diabetes, without fundus changes, and healthy controls. This data set is a valuable addition to current research, as it can be used as a reference data set for further studies using the SSOCTA Plex Elite 9000. As results from different SS-OCTA can differ significantly it is important to have this data available to be able to compare results ${ }^{12}$.

Study groups using the same SS-OCTA device as our study but different algorithms present different results among Type-2 and Type-1 diabetic patients without DR.

One group found significant quantitative changes in PD and VD in Type-1 diabetics without DR using $6 \times 6 \mathrm{~mm}$ OCTA scans and ImageJ software for analysis ${ }^{13}$. Although the group used the same device it is difficult to compare results because of different image analysis method and scan size. Regarding scan size, a study group 
found the best predictability for detecting DR using SS-OCTA is using $3 \times 3 \mathrm{~mm}$ scans ${ }^{14}$. Hence, we assume, scan size could play an important factor. In addition, the higher risk for developing DR earlier in Type-1 diabetics is well known ${ }^{15}$. Thus, diabetes Type may play an important role for the visualization of early changes in retinal microvasculature using SS-OCTA and should be considered when automated image analysis is used. Disease duration may impact results of retinal microvasculature using OCTA imaging. It is known, that prevalence for clinical DR is directly associated with disease duration. A global analysis found a prevalence of $34.6 \%$ after a median duration of 7.9 years ${ }^{15}$. Mean disease duration in our study was 5.7 years. Thus, we were able to recruit diabetics at an early stage of disease where we found no changes in PD and VD using SS-OCTA, which may further be explained by the early disease diagnosis.

Another group reported a reduction in retinal perfusion both in superficial and deep capillary plexuses with worsening DR severity and found no significant changes in VD and PD among non-DR eyes after a mean duration of 7.5 which is concordant to our results ${ }^{14}$. Previous investigations using SS-OCTA found that VD changes are associated with the presence of DR and DR severity. The group showed a negative correlation between VD and DR stage, including non-DR, indicating VD decreases with the severity of DR stage ${ }^{16}$.

Factors like disease duration and diabetes aetiology might influence retinal microvasculature and must be taken into account when automated image analysis is used for detection of DR.

Better prediction of DR was evidenced when using small scan sizes compared to widefield scans. Microvasculature damage related to DR was suggested to begin around the macula are and thus the inner $3 \mathrm{~mm}$ would be expected to have the best 
predictive sensitivity for DR. The same group found no significant change in PD and VD among eyes without clinical DR in Type-2 diabetes patients ${ }^{14}$.

Another group investigated the role of scan size regarding VD and PD changes of different DR stages and examined within the inner $5.5 \mathrm{~mm}$ of the fovea no significant changes in VD and PD among diabetics without DR compared to healthy subjects using again the same SS-OCTA device ${ }^{17}$. Regarding scan size and the previous found best predictability for finding DR changes on SS-OCTA our study focused on the inner $3 \mathrm{~mm}$, where we found no changes in PD and VD among non DR eyes.

Data of patients without clinical signs of DR is rare and not always concordant due to different OCTA devices, algorithms and at least clinical parameters like disease duration and diabetes type.

Regarding FAZ, we demonstrated no significant difference in FAZ size between patients with diabetes without DR and healthy participants. Several other studies between patients without DR and nondiabetic patients were not able to demonstrate a significant difference in FAZ size ${ }^{13,18-20}$. Literature shows variable results in qualitative descriptions of the FAZ in diabetic patients without DR. Some authors describe a clear and regular appearance of the foveal avascular zone (FAZ) borders of the superficial capillary plexus (SCP), whereas other study groups show alterations in the FAZ border ${ }^{21,22}$. Irregular FAZ borders may explain a larger falsenegative calculated FAZ area.

Our study did not find a significant difference between both groups considering irregular borders. Contrarily, other studies on a study on type-1 and type-2 diabetics without DR were able to show a significant enlarged FAZ size using SS-OCTA 4,21,22. 
The inconsistency in FAZ size may be due to the high variability of the FAZ area in healthy individuals ${ }^{23}$.

Limitations of our study include the cross- sectional design. A longitudinal analysis may reveal changes in the retinal microvasculature parameters in those patients as the risk of developing DR increases over time. The evaluation of shape and area of the FAZ, which vary consistently even in normal individuals, shows another limitation, however our results are conclusive with current literature concerning the FAZ size in patients with diabetes and without DR.

Secondly, we examined a small sample size and further studies with a larger sample size of diabetic patients are needed.

Automated image analysis is the future of retinal imaging and robust data sets are crucial. As it was demonstrated before, results from different OCTA devices is nearly impossible ${ }^{12}$. Therefor the availability of data sets for each OCTA device is beneficial to compare future results. With our study we describe a detailed complete data set, which can be used as reference data for study planning or a control group. In our data set of patients without DR we investigated no significant changes in quantitative parameters regarding microvasculature compared to healthy individuals. This data set is a valuable addition to current research, as it can be used as a reference data set for further studies using the SS-OCTA Plex Elite 9000. As results from different SS-OCTA can differ significantly it is important to have this data available to be able to compare results.

Further studies especially on patients at a very early stage of the disease are needed. Efforts should be reached in understanding early retinal microvasculature 
changes to properly detect early biomarkers as this would make a more precisely and efficient screening of DR possible, before first complications arise. 


\section{Methods}

In this prospective observational, cross-sectional study we recruited Type-2 diabetic patients without clinical signs of DR and age-matched healthy individuals visiting the University Clinic for Ophthalmology at the Kepler University Clinic between January and December 2019. The tenets of the Declaration of Helsinki were followed, approval was obtained from the National Ethics Committee for Clinical Research, and written informed consent to participate in the study was obtained from all individuals after all procedures were explained.

All patients underwent full ophthalmological examination including tonometry and fundoscopy. Patients suffering from diabetes were examined and only included if no clinical signs of diabetic retinopathy according to the International Diabetic Retinopathy Severity Scale were present ${ }^{24}$. The same procedure was applied to patients not suffering from diabetes. Exclusion criteria were any severe media opacity, ocular diseases that may affect ocular circulation like glaucoma, history of intraocular surgery and retinal laser, previous intraocular anti-VEGF/steroid treatment or significant retinal pathology that might confound results. The same exclusion criteria were applied to the healthy control group, further exclusion criteria for this group were diabetes or cardiovascular disease (history of uncontrolled hypertension, myocardial infarction, coronary heart disease or stroke). Informed consent was obtained from all study participants.

OCTA was performed using an Ultra-high-speed SS-OCT by Zeiss (Plex Elite 9000, Zeiss Medical, Dublin, CA; USA). Ss-OCTA from Zeiss uses a tunable laser with a wavelength between $1040 \mathrm{~nm}$ and $1060 \mathrm{~nm}$. It generates up to 100.000 A-scans per second with an A-scan depth of $3 \mathrm{~mm}$ in tissue and an axial resolution of $6.3 \mu \mathrm{m}$. Differences between B-scans are used to generate relative reflectivity changes with 
time secondary to motion. For each eye, fovea-centered $3 \times 3 \mathrm{~mm}$ OCTA scans were acquired because of the highest scan quality ${ }^{14}$. Low-quality OCTA images with signal strength $\leq 6$ and images with motion artefacts were excluded from the study.

Before starting quantitative analysis, all pictures were anonymized. En-face images of the superficial retinal layer (SRL), deep retinal layer (DRL) and full thickness retina images, using the built-in software segmentation algorithm, were exported. If the algorithm failed, segmentation was done manually. The SRL, extends from the inner limiting membrane to the inner plexiform layer, and the DRL, extends from the inner plexiform layer to the outer plexiform layer. Furthermore, the full-thickness slab is extending from the inner limiting membrane to $70 \mu \mathrm{m}$ above the retinal pigment epithelium. Figure 1 showes the images of a diabetic patient without DR. 


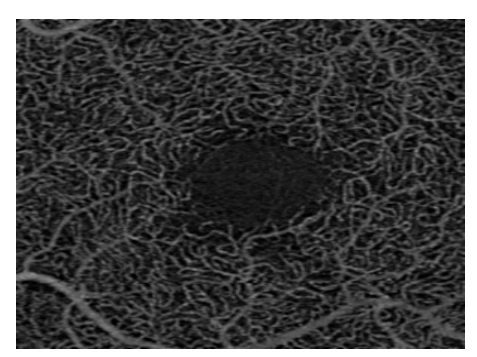

a) Angio En-face DRL

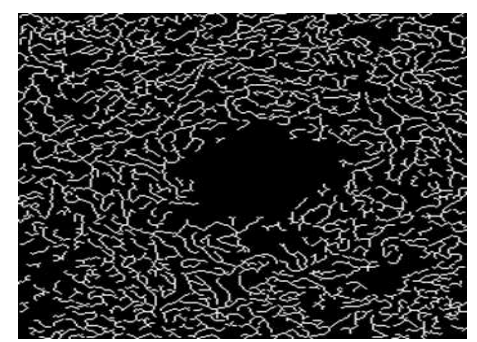

c) Vessel Trace DRL

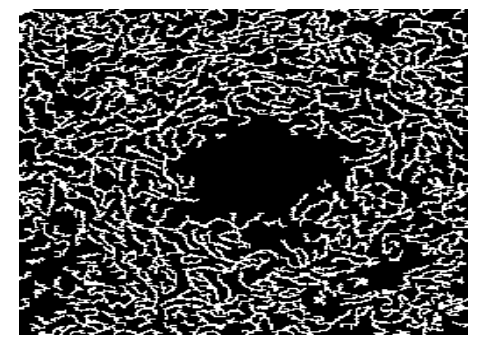

e) Perfusion Trace DRL

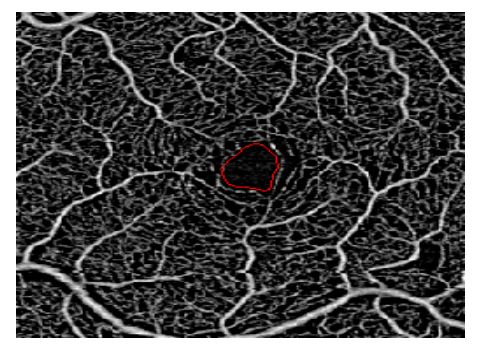

g) SRL with FAZ

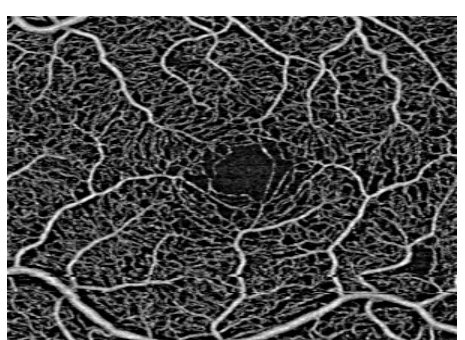

b) Angio En-face SRL

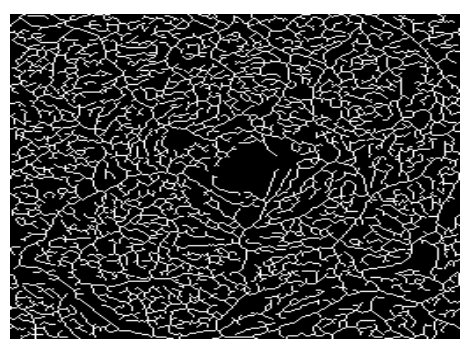

d) Vessel Trace SRL

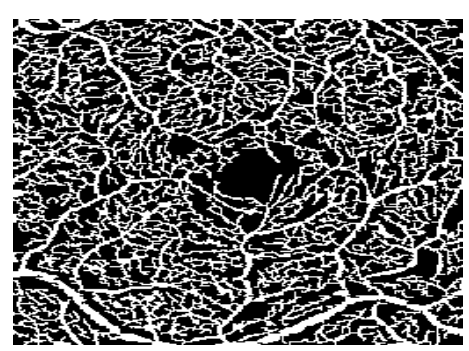

f) Perfusion Trace SRL

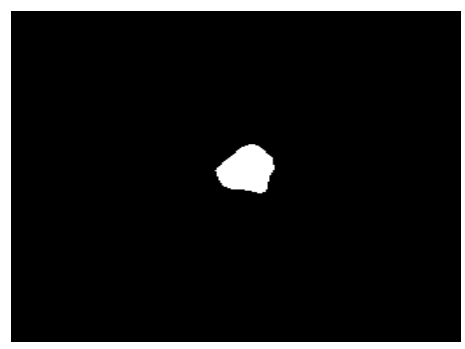

h) FAZ image

Figure 1 Optical coherence tomography angiography slabs of a diabetic patient without diabetic retinopathy. a) + b) show the Angio en Face slab of the deep and superficial retinal layer. c) $+d$ ) show the vessel density of the deep and superficial retinal layer. e) + f) demonstrate the perfusion density of the deep and superficial retinal layer. g) Superficial retinal layer and the foveal avascular zone. $h$ ) Area of the foveal avascular zone

For quantitative analysis all pictures were uploaded on the ARI Network offered by Zeiss (ARI Network portal Version 5.1), a collaboration initiative only for research use. Macular Density V 0.7 algorithm was used to investigate vessel density (VD) 
and perfusion density (PD) in the SRL and DRL. The algorithm creates slab images of the retina, the SRL and the DRL without projection artifacts. Angio images are binarized to form a black/white illustration of the vessels. To represent the PD metric, the corresponding binarized images maintain the thickness of the individual vessels, whereas for the VD calculation all vessels are shown as a thin line. PD is defined as the total area of perfused vasculature per unit area in a region of measurement. The result is a number ranging from 0 (no perfusion) to 1 (fully perfused), which is represented by pixel values ranging from 0 to 255 . Vessel Density is defined as the total length of perfused vasculature per unit area in a region of measurement. Beside these quantitative parameters also the FAZ was investigated with the algorithm mentioned above. We used the FAZ raw size of the SRL to compare it between diabetics and healthy individuals.

The primary outcome measures were the quantitative parameters VD and PD compared between the diabetic and non-diabetic group. Furthermore, FAZ size between both groups was compared.

Statistical analysis was performed using SPSS (version 21.0; SPSS Inc., Chicago, Illinois, USA). Data were confirmed to be normally distributed using Shapiro-Wilk test. To compare the data an independent, two- sampled Student's T-Test was applied. All 95\%-Confidence intervals $(95 \% \mathrm{Cl})$ and $p$-values are two-sided. Data were assumed significant with a two-sided $p<0.05$. 


\section{Declaration}

\section{Acknowledgment}

The authors would like to thank the whole team of the

Department of Ophthalmology and Optometry of the Kepler University Clinic for the achievement of this study.

\section{Conflict of Interest}

All authors report no conflict of interest.

No funding was received for conducting this study

\section{Ethics Approval}

Ethical approval was obtained by the local Ethics Committee of the Land Upper Austria and informed consent was obtained from all study participants.

\section{Data Statement}

The data that support the findings of this study are available on request from the corresponding author. The data are not publicly available due to privacy or ethical restrictions 


\section{References}

1. Whiting, D. R., Guariguata, L., Weil, C. \& Shaw, J. IDF Diabetes Atlas : Global estimates of the prevalence of diabetes for 2011 and 2030. Diabetes Res. Clin. Pract. 94, 311-321 (2011).

2. Congdon, N. G., Friedman, D. S. \& Lietman, T. Important causes of visual impairment in the world today. JAMA 290, 2057-60 (2003).

3. Dimitrova, G., Chihara, E., Takahashi, H., Amano, H. \& Okazaki, K. Quantitative Retinal Optical Coherence Tomography Angiography in Patients With Diabetes Without Diabetic Retinopathy. Investig. Opthalmology Vis. Sci. 58, 190 (2017).

4. Vujosevic, S. et al. Early Microvascular and Neural Changes in Patients With Type 1 and Type 2 Diabetes Mellitus Without Clinical Signs of Diabetic Retinopathy. Retina 1 (2017) doi:10.1097/IAE.0000000000001990.

5. Choi, W. et al. ULTRAHIGH SPEED SWEPT SOURCE OPTICAL COHERENCE TOMOGRAPHY ANGIOGRAPHY OF RETINAL AND CHORIOCAPILLARIS ALTERATIONS IN DIABETIC PATIENTS WITH AND WITHOUT RETINOPATHY. Retina 37, 11-21 (2017).

6. Grzybowski, A. et al. Artificial intelligence for diabetic retinopathy screening: a review. Eye (Basingstoke) vol. 34 451-460 (2020).

7. Schlegl, T. et al. Fully Automated Detection and Quantification of Macular Fluid in OCT Using Deep Learning. Ophthalmology 125, 549-558 (2018).

8. Wong, T. Y. \& Bressler, N. M. Artificial intelligence with deep learning technology looks into diabetic retinopathy screening. JAMA - Journal of the American Medical Association vol. 316 2366-2367 (2016).

9. Sandhu, H. S. et al. Automated diabetic retinopathy detection using optical coherence tomography angiography: A pilot study. Br. J. Ophthalmol. 102, 1564-1569 (2018).

10. Schottenhamml, J. et al. An automatic, intercapillary area-based algorithm for quantifying diabetes-related capillary dropout using optical coherence tomography 
angiography. in Retina vol. 36 S93-S101 (Lippincott Williams and Wilkins, 2016).

11. Govindaswamy, N. et al. Vascular changes precede tomographic changes in diabetic eyes without retinopathy and improve artificial intelligence diagnostics. J. Biophotonics 13, (2020).

12. Corvi, F. et al. Reproducibility of Vessel Density, Fractal Dimension, and Foveal Avascular Zone Using 7 Different Optical Coherence Tomography Angiography Devices. Am. J. Ophthalmol. 186, 25-31 (2018).

13. Vujosevic, S. et al. Early Detection of Microvascular Changes in Patients with Diabetes Mellitus without and with Diabetic Retinopathy : Comparison between Different Swept-Source OCT-A Instruments. 2019, (2019).

14. Hirano, T. et al. Quantifying vascular density and morphology using different sweptsource optical coherence tomography angiographic scan patterns in diabetic retinopathy. Br. J. Ophthalmol. bjophthalmol-2018-311942 (2018) doi:10.1136/bjophthalmol-2018-311942.

15. Yau, J. W. Y. et al. Global prevalence and major risk factors of diabetic retinopathy. Diabetes Care 35, 556-564 (2012).

16. Xie, N. et al. Macular vessel density in diabetes and diabetic retinopathy with sweptsource optical coherence tomography angiography. Graefe's Arch. Clin. Exp. Ophthalmol. 258, 2671-2679 (2020).

17. Mastropasqua, R. et al. Widefield optical coherence tomography angiography in diabetic retinopathy. Acta Diabetol. 56, 1293-1303 (2019).

18. Simonett, J. M. et al. Early microvascular retinal changes in optical coherence tomography angiography in patients with type 1 diabetes mellitus. 751-755 (2017) doi:10.1111/aos.13404.

19. Goudot, M. M. et al. Parafoveal OCT Angiography Features in Diabetic Patients without Clinical Diabetic Retinopathy: A Qualitative and Quantitative Analysis. J. Ophthalmol. 2017, (2017).

20. Cao, D. et al. Optical coherence tomography angiography discerns preclinical diabetic 
retinopathy in eyes of patients with type 2 diabetes without clinical diabetic retinopathy. Acta Diabetol. (2018) doi:10.1007/s00592-018-1115-1.

21. Gong, D. et al. A morphological study of the foveal avascular zone in patients with diabetes mellitus using optical coherence tomography angiography. Graefe`s Arch. Clin. Exp. Ophthalmol. 254, 873-9 (2016).

22. de Carlo, T. E. et al. DETECTION OF MICROVASCULAR CHANGES IN EYES OF PATIENTS WITH DIABETES BUT NOT CLINICAL DIABETIC RETINOPATHY USING OPTICAL COHERENCE TOMOGRAPHY ANGIOGRAPHY. Retina 35, 2364-70 (2015).

23. Gómez-Ulla, F. et al. Age and gender influence on foveal avascular zone in healthy eyes. Exp. Eye Res. 189, (2019).

24. Wilkinson, C. P. et al. Proposed international clinical diabetic retinopathy and diabetic macular edema disease severity scales. Ophthalmology 110, 1677-82 (2003). 


\section{Figure Legends}

Figure 1 Optical coherence tomography angiography slabs of a diabetic patient without diabetic retinopathy. a) $+b$ ) show the Angio en Face slab of the deep and superficial retinal layer. c) $+d$ ) show the vessel density of the deep and superficial retinal layer. e) +f) demonstrate the perfusion density of the deep and superficial retinal layer. g) Superficial retinal layer and the foveal avascular zone. h) Area of the foveal avascular zone 


\section{Figures}

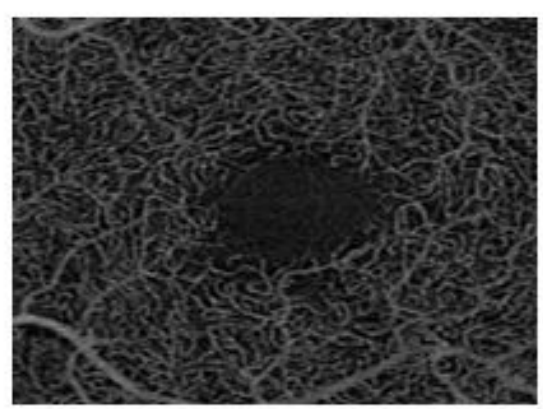

a) Angio En-face DRL

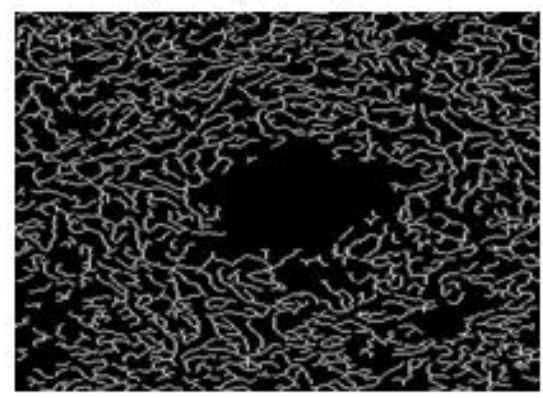

c) Vessel Trace DRL

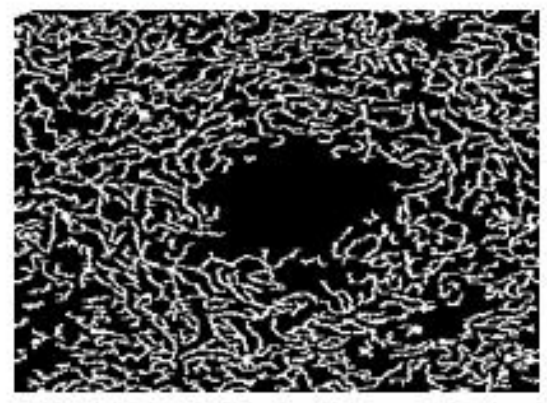

e) Perfusion Trace DRL

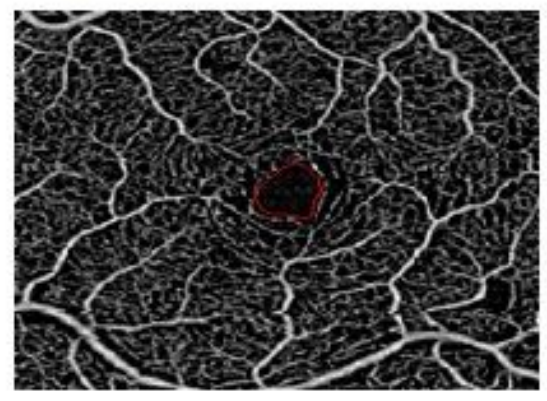

g) SRL with FAZ

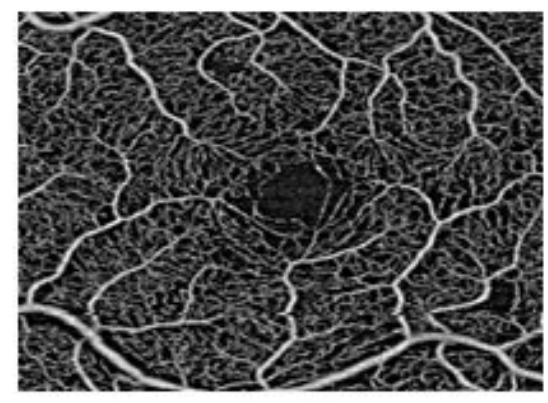

b) Angio En-face SRL

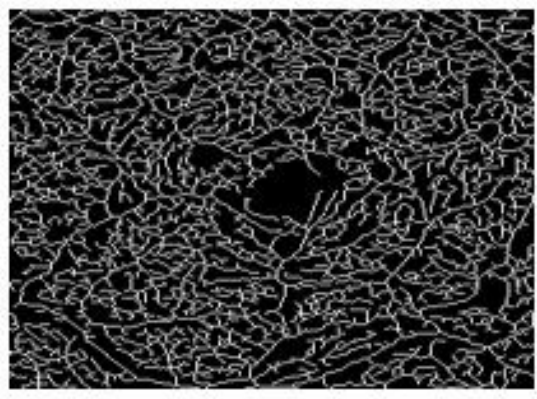

d) Vessel Trace SRL

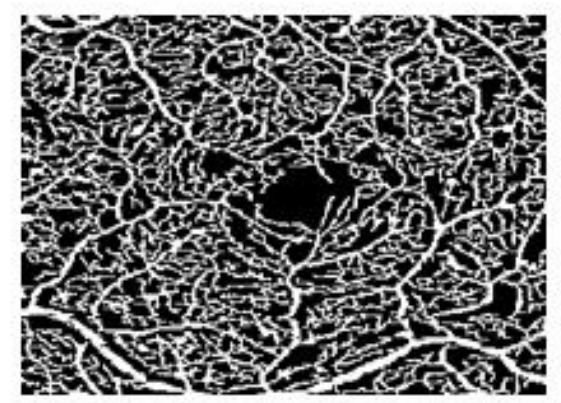

f) Perfusion Trace SRL

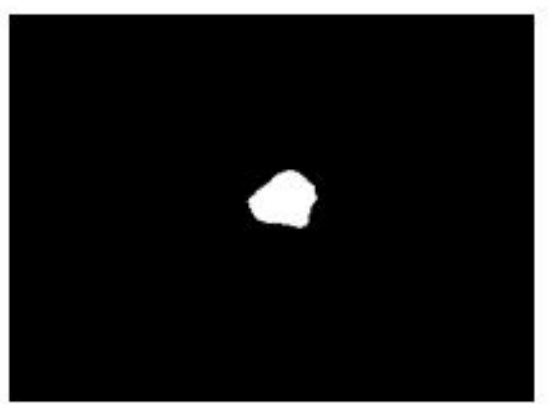

h) FAZ image

\section{Figure 1}

Optical coherence tomography angiography slabs of a diabetic patient without diabetic retinopathy. a) + b) show the Angio en Face slab of the deep and superficial retinal layer. c) + d) show the vessel density of the deep and superficial retinal layer. e) $+f$ ) demonstrate the perfusion density of the deep and superficial 
retinal layer. g) Superficial retinal layer and the foveal avascular zone. h) Area of the foveal avascular zone 